quent hybridization using the same microarray panel. The results show that the overall changes in this tumor amount to about $16 \%$ of the $\mathrm{CpG}$ islands analyzed, with $8.2 \%$ of these loci showing changes in copy number, $6.1 \%$ showing changes in methylation status and $1.6 \%$ showing both types of changes. We have further categorized these genetic and epigenetic changes into eight distinct classes. Selected clones from each class have been confirmed by Southern hybridization. This study is the first such detailed analysis of complex molecular alterations in a single tumor, and it demonstrates the potential utility of this $\mathrm{CpG}$ island microarray for tumor classification.

Huminiecki, Lukasz

\section{In silico cloning of new endothelial-specific genes}

Lukasz Huminiecki \& Roy Bicknell

ICRF, IMM, JR Hospital, Oxford University, UK

We present a powerful new approach for comparative expression analysis combining two data-mining strategies followed by experimental verification. The endothelium plays a pivotal role in many physiological and pathological processes and is known to be an exceptionally active transcriptional site. To advance our understanding of endothelial cell biology and to elucidate potential pharmaceutical targets, we have developed a new database screening approach to permit identification of new endothelial-cell-specific genes. We screened the UniGene index using high-stringency BLAST against a pool of endothelial and a pool of nonendothelial expressed sequence tags constructed from cell-type-specific db expressed sequence tag libraries. UniGene clusters with matches in the endothelial pool and no matches in the non-endothelial pool were selected. We then combined this approach with Serial Analysis of Gene Expression-library subtraction and the polymerase chain reaction with reverse transcription to further examine clusters of interest. We identified and labeled four new genes: those coding for endothelialcell-specific molecules (ECSM) 1-3 and magic roundabout (similar to the axon guidance protein roundabout)

Ignatovich, Irina A.

\section{Suicide effect of herpesvirus thymidine kinase gene delivered into human hepatoma cells in complex with oligopeptides}

Irina A. Ignatovich, Ella B. Dizhe, Sergej V. Burov,

Boris N. Akifiev \& Andrej A. Perevozchikov

Institute of Experimental Medicine, Acad. Pavlova Street 12, St. Petersburg 197376, Russia

The delivery of the suicide herpes simplex virus type 1 thymidine kinase (TK) gene into tumor cells followed by therapy with synthetic guanylic nucleotide analogues, such as gancyclovir and acyclovir, is one approach to cancer treatment. The most effective strategy for suicide gene therapy is virus-mediated TK gene transfer, using mainly recombinant adenoviruses or retroviruses. The main disadvantages of this strategy include immunogenicity, toxicity (for adenoviral vectors) and the risk of side effects (for retroviral vectors). An alternative approach involves the use of nonviral gene delivery systems, such as molecular conjugates, cationic oligopeptides and cationic lipids. Although these are less effective than viral vectors, they lack the above-mentioned disadvantages and may be used repeatedly. To evaluate the possibility of in vivo nonviral delivery of the herpesvirus TK gene into mam- malian tumors, we have studied the antitumor effect of this gene after its transfer into human hepatoma (HepG2) cells in complex with cationic oligopeptides, followed by acyclovir treatment. The herpesvirus TK gene was under the control of the mouse ribosomal L32 protein promoter, which is active only in proliferating cells. We used a gene transfer system based on the cationic peptide YKAK8WK, which is capable of forming compact complexes with plasmid DNA, and the amphipatic peptide JTS-1, a pH-dependent, endosome-destabilizing agent. TKgene-transduced cells were cultivated in growth medium containing acyclovir (50 $\mu \mathrm{g} \mathrm{ml}^{-1}$ ). The percentage of dead cells was estimated after $48 \mathrm{~h}$ by propidium iodine staining. The apoptotic death of cells was simultaneously confirmed by gel electrophoresis detection of oligonucleosomic DNA fragments. The amount of dead cells among the HepG2 cells transfected by suicide TK genes was four- to fivefold more than the amount among control HepG2 cells grown at the same concentration of acyclovir in medium. These results demonstrate the possibility of using cationic oligopeptides as suicide TK gene carriers in gene therapeutic treatments of human tumors.

Inoue, Norimitsu

[17]

\section{Determination of the breakpoints of a chromosomal translocation found in a patient with paroxysmal nocturnal hemoglobinuria}

Norimitsu Inoue ${ }^{1}$, Tomohisa Izui ${ }^{1}$, Maki Kuwayama ${ }^{1,2}$ Junichi Nishimura ${ }^{1,2}$, Ken Kurokawa $^{3}$, Takashi Machii ${ }^{2}$ Yuzuru Kanakura ${ }^{2} \&$ Taroh Kinoshita $^{1}$

${ }^{1}$ Research Institute for Microbial Diseases, Osaka University, Osaka, Japan ${ }^{2}$ Department of Hematology and Oncology, Osaka University, Osaka, Japan ${ }^{3}$ Genome Information Research Institute, Osaka University, Osaka, Japan

Paroxysmal nocturnal hemoglobinuria is an acquired hematopoietic stem cell disorder that causes clonal expansion of glycosylphosphatidylinositol (GPI) anchor-deficient cells. A somatic mutation of PIG-A that encodes a subunit of the enzyme complex in GPI anchor synthesis results in deficiency of GPI-anchored proteins on the surface of the blood cell. The mechanisms of clonal expansion of abnormal hematopoietic stem cells are unclear. We found a patient with the disorder having chromosomal abnormality $46 \mathrm{XX}, \mathrm{t}(12 ; 12)(\mathrm{q} 13 ; \mathrm{q} 15)$ in all cells bearing a mutation of PIG-A and only in those cells, suggesting that the translocation may be causally related to clonal expansion. We mapped the breakpoints to clone genes that may be involved in the pathogenesis of this disease. Because the patient did not have GPI anchor-deficient lymphocytes, it was difficult to establish GPI anchor-deficient cell lines. We constructed somatic cell hybrids by fusing GPI anchor-deficient monocytes to mouse myeloma cells deficient in hypoxanthine phosphoribosyltransferase and selected hybrids carrying chromosome 12 by monitoring the expression of human CD9. We chose hybrids carrying chromosome 12 with deletion or duplication of the q13-q15 region by analyzing microsatellite markers. We mapped the breakpoints between WI-9630 and CHLC.ATA29H01 in q13 and between D12S355 and sts-N34486 in q15 using the hybrids carrying the deleted chromosome 12 . We designed new sequence tagged site markers around D12S355 and fine-mapped the breakpoint in q15 within a distance of 2 kilobase pairs. The breakpoint in q13 was determined by the inverse polymerase chain reaction using primers near the breakpoint in q15. 